\title{
On the zeros of Meixner polynomials
}

\author{
A Jooste · K Jordaan • F Toókos
}

Received: date / Accepted: date

\begin{abstract}
We investigate the zeros of a family of hypergeometric polynomials $M_{n}(x ; \beta, c)=(\beta)_{n}{ }_{2} F_{1}\left(-n,-x ; \beta ; 1-\frac{1}{c}\right), n \in \mathbb{N}$, known as Meixner polynomials, that are orthogonal on $(0, \infty)$ with respect to a discrete measure for $\beta>0$ and $0<c<1$. When $\beta=-N, N \in \mathbb{N}$ and $c=\frac{p}{p-1}$, the polynomials $K_{n}(x ; p, N)=(-N)_{n}{ }_{2} F_{1}\left(-n,-x ;-N ; \frac{1}{p}\right), n=0,1, \ldots N, 0<p<1$ are referred to as Krawtchouk polynomials. We prove results for the zero location of the orthogonal polynomials $M_{n}(x ; \beta, c), c<0$ and $n<1-\beta$, the quasiorthogonal polynomials $M_{n}(x ; \beta, c),-k<\beta<-k+1, k=1, \ldots, n-1$ and $0<c<1$ or $c>1$, as well as the polynomials $K_{n}(x ; p, N)$ with non-Hermitian orthogonality for $0<p<1$ and $n=N+1, N+2, \ldots$. We also show that the polynomials $M_{n}(x ; \beta, c), \beta \in \mathbb{R}$ are real-rooted when $c \rightarrow 0$.
\end{abstract}

Keywords Zeros · Orthogonal polynomials · Quasi-orthogonal polynomials · Real-rooted polynomials · Meixner polynomials $\cdot$ Krawtchouk polynomials

Mathematics Subject Classification (2000) 33C45 $\cdot 42 \mathrm{C} 05$

Research by K Jordaan was partially supported by the National Research Foundation under grant number 2054423.

\footnotetext{
A Jooste

Department of Mathematics and Applied Mathematics, University of Pretoria, Pretoria, 0002, South Africa

E-mail: alta.jooste@up.ac.za

K Jordaan

Department of Mathematics and Applied Mathematics, University of Pretoria, Pretoria, 0002, South Africa

Tel.: +27124202788

Fax: +27124203893

E-mail: kjordaan@up.ac.za

F Toókos

Institute for Biomathematics and Biometry, Helmholtz Zentrum München, Neuherberg, Germany

E-mail: ferenc.tookos@helmholtz-muenchen.de
} 


\section{Introduction}

Polynomials that are real-rooted are of interest in various branches of mathematics, including combinatorics and approximation theory. Real-rootedness is used to prove the log-concavity and unimodality of the sequence of coefficients of a polynomial which otherwise demands cumbersome combinatorial manipulations (see e.g. [33] and [7]) and is useful in establishing convergence properties of rational approximants (cf. [16]).

The real-rootedness of a given polynomial is a classical problem and various tools are available to prove that the zeros are real, for example, in [6] and [17], Pólya frequency sequences are used to show that some hypergeometric polynomials have only real roots. One of the standard ways to prove realrootedness of polynomials, is to use their relation to orthogonal polynomials. A sequence of polynomials $\left\{P_{n}\right\}_{n=0}^{N}, N \in \mathbb{N} \cup\{\infty\}$ is orthogonal with respect to some positive Borel measure $d \phi$, if

$$
\int_{S} P_{n}(x) P_{m}(x) d \phi(x)=d_{n}^{2} \delta_{m n}, m, n=0,1, \ldots N
$$

where $S$ is the support of the measure and $\delta_{m n}$ is Kronecker's symbol. It is a well-known classical result that the $n$ zeros of $P_{n}$ are real, distinct and lie in the convex hull of $\operatorname{supp}(\phi)$ (cf. [35]).

If the measure $d \phi$ is discrete and $\left(\rho_{i}\right)$ are the values of the weight at the distinct points $\left\{x_{i}\right\}_{i=0}^{N-1}$, then (1) becomes (cf. [5])

$$
\sum_{i=0}^{N-1} P_{n}\left(x_{i}\right) P_{m}\left(x_{i}\right) \rho_{i}=d_{n}^{2} \delta_{m n}, m, n=0,1, \ldots N
$$

and the sequence $\left\{P_{n}\right\}_{n=0}^{N}$ is discrete orthogonal.

The classical orthogonal polynomials of a discrete variable are used extensively and have many applications in various fields including combinatorial analysis, theoretical and mathematical physics, group representation theory and stochastic processes. For example, Meixner polynomials have been used to analyse discrete stochastic processes in the context of spectral analysis in the Laplace domain (cf. [18]) and close relationships have been found linking generalised spherical harmonics for $\mathrm{SU}(2)$ with a special class of Meixner polynomials known as Krawtchouk polynomials [25].

In this paper we make a comprehensive study of the zero location of Meixner and Krawtchouk polynomials, in particular for parameter values where (some of) the zeros are real.

Meixner polynomials are defined in terms of the ${ }_{2} F_{1}$ hypergeometric function (cf. $[24,(9.10 .1)])$

$$
\begin{aligned}
M_{n}(x ; \beta, c) & =(\beta)_{n}{ }_{2} F_{1}\left(-n,-x ; \beta ; 1-\frac{1}{c}\right) \\
& =(\beta)_{n} \sum_{k=0}^{n} \frac{(-n)_{k}(-x)_{k}\left(1-\frac{1}{c}\right)^{k}}{(\beta)_{k} k !}, \beta, c \in \mathbb{R}, c \neq 0
\end{aligned}
$$


where $\beta \neq-1,-2, \ldots,-n+1$ and ( $)_{n}$ is Pochhammer's symbol defined by

$$
\begin{aligned}
& (\alpha)_{n}=(\alpha)(\alpha+1) \ldots .(\alpha+n-1) \text { for } n \geq 1 \\
& (\alpha)_{0}=1 \text { when } \alpha \neq 0 .
\end{aligned}
$$

The sequence $\left\{M_{n}(x ; \beta, c)\right\}_{n=0}^{\infty}$ satisfies the discrete orthogonality relation (cf. $[24,(9.10 .2)])$

$$
\sum_{x=0}^{\infty} \frac{c^{x}(\beta)_{x}}{x !} M_{m}(x ; \beta, c) M_{n}(x ; \beta, c)=\frac{(\beta)_{n} n !}{c^{n}(1-c)^{\beta}} \delta_{m n}
$$

when $0<c<1$ and $\beta>0$, and hence the zeros are real, distinct and lie in $(0, \infty)$ for these values of the parameters $\beta$ and $c$. It can also be shown that, for the same parameter values, the Meixner polynomials have a non-standard orthogonality property defined in terms of a discrete inner product involving difference operators (cf. [1]).

When $c>1$ and $\beta>0$ the orthogonality relation (3) can be written as (cf. $[10$, p.177 (3.7)])

$$
\sum_{k=0}^{\infty} \frac{(\beta)_{k}}{c^{k} k !} M_{m}(-k-\beta ; \beta, c) M_{n}(-k-\beta ; \beta, c)=\frac{(\beta)_{n} c^{\beta} n !}{c^{n}(c-1)^{\beta}} \delta_{m n}
$$

by an application of the Pfaff-Kummer transformation (cf. [24, (1.7.2)]) and therefore the zeros of the polynomials $\left\{M_{n}(x ; \beta, c)\right\}_{n=0}^{\infty}$ are distinct and in $(-\infty,-\beta)$.

A non-Hermitian orthogonality with respect to a complex weight function for Meixner polynomials $M_{n}(x ; \beta, c)$ when $\beta, c \in \mathbb{C}, c \notin[0, \infty)$ and $\beta \notin \mathbb{Z}^{-}$is discussed in [12, Proposition 9]. The standard orthogonality for a finite number of Meixner polynomials $M_{n}(x ; \beta, c)$ when $c<0$ and $\beta$ is equal to a negative integer, say $\beta=-N, N \in \mathbb{N}$ is that of the Krawtchouk polynomials defined by (cf. $[24,(9.11 .1)])$

$$
K_{n}(x ; p, N)=(-N)_{n}{ }_{2} F_{1}\left(-n,-x ;-N ; \frac{1}{p}\right), n=0,1, \ldots, N .
$$

The orthogonality relation for Krawtchouk polynomials is given by

$$
\sum_{x=0}^{N} w(x ; p, N) K_{m}(x ; p, N) K_{n}(x ; p, N)=0
$$

when $m<n \leq N ; m, n, N \in \mathbb{N}$ and $0<p<1$ where

$$
w(x ; p, N)=\left(\begin{array}{c}
N \\
x
\end{array}\right) p^{x}(1-p)^{N-x}
$$

is positive at the mass points $x=0,1, \ldots, N$ of the discrete measure. This implies that for $0<p<1$ and $n \leq N, n, N \in \mathbb{N}$, the zeros of $K_{n}(x ; p, N)$ are real, distinct and in the interval $(0, N)$. Furthermore, by an argument due to $\mathrm{L}$. 
Fejér there is at most one zero of $K_{n}(x ; p, N)$ in between any two consecutive mass points (cf. [22]) and in the particular case where $n=N$, the zeros of $K_{n}(x ; p, n)$, denoted by $x_{i}, i=1,2, \ldots, n$ in ascending order, interlace with the mass points as follows

$$
0<x_{1}<1<x_{2}<2<\cdots<x_{n}<n
$$

The zero asymptotics of normalised Krawtchouk polynomials when the ratio of parameter $n / N \rightarrow \alpha$ as $n, N \rightarrow \infty$ was investigated in [14] and [15] by finding the support and density of the constrained extremal measure for all possible values of the parameter $\alpha$ and the asymptotic zero distribution of Meixner polynomials has also been studied by various authors (cf. [2], [21] and [26]). The distribution of the zeros of multiple Meixner polynomials is discussed in [32].

Since $(\beta+k)_{n-k}=\frac{(\beta)_{n}}{(\beta)_{k}},(4)$ can be rewritten as

$$
K_{n}(x ; p,-\beta)=\sum_{k=0}^{n} \frac{(-n)_{k}(-x)_{k}(\beta+k)_{n-k}}{k ! p^{k}}
$$

which is valid for every $n$ and can be used to define Krawtchouk polynomials for any $n \in \mathbb{N}$ and $\beta \in \mathbb{R}$.

For $\beta \neq 0,-1, \ldots, 1-n$ we obtain

$$
\begin{aligned}
& M_{n}(x ; \beta, c)=K_{n}\left(x ; \frac{c}{c-1},-\beta\right) \\
& =(\beta)_{n}{ }_{2} F_{1}\left(-n,-x ; \beta ; 1-\frac{1}{c}\right) \\
& =(\beta)_{n}\left(\frac{1}{c}\right)^{n}{ }_{2} F_{1}(-n, x+\beta ; \beta ; 1-c) \quad(\text { cf. }[24,(1.7 .2)]) \\
& =\left(\frac{1}{c}\right)^{n} M_{n}\left(-x-\beta ; \beta, \frac{1}{c}\right)
\end{aligned}
$$

a general symmetry property of the Meixner polynomials, since by continuity it holds for $\beta \in \mathbb{R}$. Special cases of this symmetry property were proved for $\beta=N, N \in \mathbb{N}$ and $x=0, \ldots, N$ (cf. [9], [20]) using the generating function for Krawtchouk polynomials (cf. [24]) and for $x=0, \ldots, n$ in [10].

The polynomials $M_{n}(x ; \beta, c)$ have the standard orthogonality of Meixner and Krawtchouk polynomials for the parameter ranges

$$
\begin{aligned}
& 0<c<1, \beta>0, n=0,1,2, \ldots \\
& c>1, \beta>0, n=0,1,2, \ldots \text { and } \\
& c<0, \beta=-N, N \in \mathbb{N}, n=0,1,2 \ldots N
\end{aligned}
$$

hence our discussion will focus on the zeros of polynomials $M_{n}(x ; \beta, c)$ for parameter values 
(i) $c<0, \beta<0, n<1-\beta, n \in \mathbb{N}$;

(ii) $0<c<1, \beta<0, n \in \mathbb{N}$;

(iii) $c>1, \beta<0, n \in \mathbb{N}$;

(iv) $c<0, \beta=-N, n=N+1, N+2, \ldots$;

(v) $c<0, \beta>0, n \in \mathbb{N}$ and

(vi) $c \rightarrow 0, \beta \in \mathbb{R}, n \in \mathbb{N}$.

We begin with case (i) in Section 2 where we extend the conclusion following from the discrete orthogonality of Krawtchouk polynomials $K_{n}(x ; p, N)$ for integer values of the parameter $N, N+1>n, 0<p<1$ to prove that, for $c=\frac{p}{p-1}$, the zeros of the polynomials $M_{n}(x ; \beta, c)$ are real, distinct and lie in the interval $(0,-\beta)$ for all real values of the parameter $\beta, n<1-\beta$ and $c<0$. In Section 3 we consider cases (ii) and (iii), proving that the Meixner polynomials $M_{n}(x ; \beta, c)$, are quasi-orthogonal of order $k$ for $-k<\beta<-k+1$, $k=1, \ldots, n-1$ and $0<c<1$ or $c>1$.

We discuss case (iv) and (v) in Section 4. Results obtained in [3], [4], [19], [34] and [35] for the zeros of Krawtchouk polynomials $K_{n}(x ; p, N), 0<p<1$ of degree $n=N+1$, are extended to polynomials of degree $n=N+2$ and $n=N+3$. We make use of the product decomposition (cf. [12])

$$
K_{n}(x ; p, N)=K_{N+1}(x ; p, N) M_{n-N-1}\left(x-N-1 ; N+2, \frac{p}{p-1}\right)
$$

for $p \neq 0,1, n>N \in \mathbb{N}$ which also shows that for case (iv) it suffices to study polynomials $M_{n}(x ; N, c)$ for $c<0, N=1,2, \ldots$ (case (v) for integer values of $\beta)$.

In the last section we prove that the polynomials $\left\{M_{n}(x ; \beta, c)\right\}_{n=0}^{\infty}$ are realrooted for all $\beta \in \mathbb{R}$ when $c \rightarrow 0$ (case (vi)).

We observe that for the special case when $c \rightarrow \infty$, the polynomials $M_{n}(x ; \beta, c)$ tend to

$$
(\beta)_{n}{ }_{2} F_{1}(-n,-x ; \beta ; 1)=(x+\beta)(x+\beta+1) \ldots . .(x+\beta+n-1)
$$

which vanishes when $x=-\beta,-\beta-1, \ldots,-\beta-n+1$, whereas when $c \rightarrow 1$ the polynomial $M_{n}(x ; \beta, c)$ tends to

$$
(\beta)_{n}{ }_{2} F_{1}(-n,-x ; \beta ; 0)=(\beta)_{n}
$$

and has $n$ zeros at infinity if it is considered as a polynomial of degree $n$ in $x$ (cf. $[35,(6.72 .3)])$.

\section{The zeros of $M_{n}(x ; \beta, c), c<0$ and $n<1-\beta$}

The three term recurrence relation (cf. $[24,(9.10 .3)])$ for the Meixner polynomials is

$$
x M_{n}(x ; \beta, c)=A_{n} M_{n+1}(x ; \beta, c)+B_{n} M_{n}(x ; \beta, c)+C_{n} M_{n-1}(x ; \beta, c) .
$$


where $A_{n}=\frac{c}{c-1}$ and $C_{n}=\frac{n(\beta+n-1)}{c-1}$. This relation holds true for all $\beta, c \in$ $\mathbb{R}, c \neq 0,1$ and $n \in \mathbb{N}$, because it follows from a contiguous relation for hypergeometric functions (cf. [31, p.71 (6)]). For the particular case when $\beta, c<0$, we will have $A_{n-1} C_{n}>0$ when $n<1-\beta$.

It follows from a theorem often attributed to Favard (cf. [10]) that there is at least one positive measure $d \alpha(x)$ so that, for $\beta, c<0$

$$
\int_{-\infty}^{\infty} M_{n}(x ; \beta, c) M_{m}(x ; \beta, c) d \alpha(x)=0, m \neq n, n=0,1, \ldots,-\lfloor\beta\rfloor
$$

and hence $\left\{M_{n}(x ; \beta, c)\right\}_{n=0}^{-\lfloor\beta\rfloor}$ has $n$ real, distinct zeros when $\beta, c<0$. However, the set containing the real zeros does not follow immediately.

We prove that the zeros of $M_{n}(x ; \beta, c)$ for $n<1-\beta, \beta, c<0$ are in $(0,-\beta)$, by using a generalised Sturmian sequence argument applied to solutions of difference equations (cf. [30]), as was done in [28] for Hahn polynomials. We begin by proving that if $r$ denotes a zero of $M_{n}(x ; \beta, c)$ in $(0,-\beta)$, then $r-1$ and $r+1$ cannot be zeros of $M_{n}(x ; \beta, c)$ and, in addition, there will be an odd number of zeros of $M_{n}(x ; \beta, c)$ in the interval $(r-1, r+1)$.

Lemma 1 Let $\beta \in \mathbb{R}, n \in \mathbb{N}, n<1-\beta$ and $c<0$. If $r$ is a zero of $M_{n}(x ; \beta, c)$ and $r \in(0,-\beta)$, then $M_{n}(r-1 ; \beta, c) M_{n}(r+1 ; \beta, c)<0$.

Proof Let $\beta \in \mathbb{R}, n<1-\beta$ and $c<0$. Consider the difference equation (cf. $[24,(9.10 .5)])$

$$
A(x) M_{n}(x+1 ; \beta, c)+C(x) M_{n}(x-1 ; \beta, c)=B(x) M_{n}(x ; \beta, c)
$$

where $A(x)=c(x+\beta), B(x)=n(c-1)+x+(x+\beta) c$ and $C(x)=x$. Note that $A(x)>0$ and $C(x)>0$ when $x \in(0,-\beta)$ and $c<0$.

Suppose $r$ is a zero of $M_{n}(x ; \beta, c)$ in the interval $(0,-\beta)$, then

$$
A(r) M_{n}(r+1 ; \beta, c)+C(r) M_{n}(r-1 ; \beta, c)=0 .
$$

Assume that

$$
M_{n}(r+1 ; \beta, c)=0 .
$$

Letting $x=r+1$ in (12) we obtain $A(r+1) M_{n}(r+2 ; \beta, c)=0$ and if $r+1 \in$ $(0,-\beta)$, it follows that $A(r+1)>0$ and $M_{n}(r+2 ; \beta, c)=0$. By repeating this argument we can prove that

$$
M_{n}(r+i ; \beta, c)=0 \text { for all } i \text { such that } 0<r+i-1<-\beta .
$$

Under our assumption (14), it also follows from equation (13) that $C(r) M_{n}(r-1 ; \beta, c)=0$ if $r \in(0,-\beta)$ and since $C(r)>0$ for these values of $r$, we have that $M_{n}(r-1 ; \beta, c)=0$. In the same way as before we can prove that

$$
M_{n}(r-j ; \beta, c)=0 \text { for all } j \text { such that } 0<r-j+1<-\beta .
$$

In short, it follows from results (15) and (16) that $M_{n}(x ; \beta, c)$ has as zeros all numbers $r+i, i \in \mathbb{Z}$ with $-1<r+i<1-\beta$. This means that $M_{n}(x ; \beta, c)$ has 
a total of at most $\lfloor 1-\beta-(-1)\rfloor=1+\lfloor 1-\beta\rfloor \geq n+1>n$ zeros unless both $\beta$ and $r$ are integers. In this case $M_{n}(x ; \beta, c)$ has $1-\beta-(-1)-1=1-\beta>n$ zeros. In both cases, the number of zeros is greater than the degree of the polynomial and we have a contradiction. This means $M_{n}(r+1 ; \beta, c) \neq 0$.

The proof that $M_{n}(r-1 ; \beta, c) \neq 0$ is analogous. Now (13) implies that $M_{n}(r+1 ; \beta, c)=-\frac{C(r)}{A(r)} M_{n}(r-1 ; \beta, c)$ and clearly $M_{n}(r+1 ; \beta, c)$ and $M_{n}(r-1 ; \beta, c)$ differ in sign.

Theorem 2 Let $n$ be a positive integer. For any real number $\beta, n<1-\beta$, and $c<0$, the zeros of $M_{n}(x ; \beta, c)$ lie in the open interval $(0,-\beta)$.

Proof Let $n<1-\beta, c<0$ and let $n$ and $N$ be integers, such that $N=\lceil-\beta\rceil$ where $\lceil a\rceil$ denotes the least integer larger than or equal to $a$. In the sequence

$$
M_{n}(0 ; \beta, c), M_{n}(1 ; \beta, c), \ldots \ldots, M_{n}(N ; \beta, c)
$$

each term can be considered as a polynomial function of the parameter $\beta$ with $c<0$ fixed. When a numerical value is assigned to $\beta$, we denote the number of variations in sign in the resulting sequence by $V(\beta)$. We want to determine $V(\beta)$ for $N-1<-\beta \leq N$.

When $-\beta=N$, it follows from (5) and (7) that the sequence of polynomials in (17) will have $n$ sign changes since $K_{n}\left(x ; \frac{c}{c-1}, N\right)$ is orthogonal for $c<0$. This means that $V(-N)=n$.

If $-\beta$ is assigned any value in the interval $(N-1, N]$, then Lemma 1 implies that in the resulting sequence

$$
M_{n}(0 ; \beta, c), M_{n}(1 ; \beta, c), \ldots \ldots, M_{n}(N ; \beta, c)
$$

no two consecutive terms are zero and also that if $M_{n}(i ; \beta, c)=0$ for $i=$ $1,2, \ldots N-1$, then the two adjacent terms: $M_{n}(i-1 ; \beta, c)$ and $M_{n}(i+1 ; \beta, c)$ differ in sign. Moreover, it follows directly from (2) that

$$
M_{n}(0 ; \beta, c)=(\beta)_{n}
$$

and the first term can never be zero for $-\beta$ in the interval $(N-1, N]$. The last term does not change sign on $(N-1, N]$ since by $(8)$

$$
M_{n}(N ; \beta, c)=(\beta)_{n}\left(\frac{1}{c}\right)^{n} \sum_{i=0}^{n} \frac{(-n)_{i}(\beta+N)_{i}(1-c)^{i}}{(\beta)_{i} i !}>0
$$

for all $-\beta \in(N-1, N]$.

These conditions are sufficient to ensure that the sequence (17) forms a generalised Sturmian sequence and therefore $V(\beta)$ remains constant as $-\beta$ increases through the interval $(N-1, N]$. Hence $V(\beta)=n$ for all $-\beta \in(N-1, N]$.

Thus for $n<1-\beta, M_{n}(x ; \beta, c)$ changes sign $n$ times for $x$ in $(0, N)$ and since the degree is $n$ we conclude that $M_{n}(x ; \beta, c)$ has $n$ distinct roots in $(0, N)$.

If $r$ is a root of $M_{n}(x ; \beta, c)$, then $0<r<N$ and it follows from relation (9) that $-\beta-r$ will be a zero of $M_{n}\left(x ; \beta, \frac{1}{c}\right)$ with $0<-\beta-r<N$, i.e. $r<-\beta$. We conclude that the zeros of $M_{n}(x ; \beta, c)$ are in the open interval $(0,-\beta)$. 
3 Quasi-orthogonality of $M_{n}(x ; \beta, c), \beta<0$ and $0<c<1$ or $\beta<0$ and $c>1$

A polynomial $P_{n}$ of exact degree $n \geq r$, is quasi-orthogonal of order $r$ on $[a, b]$ with respect to a weight function $w(x)>0$, if (cf. [8, p.159])

$$
\int_{a}^{b} x^{j} P_{n}(x) w(x) d x\left\{\begin{array}{l}
=0, \text { for } j=0,1, \ldots, n-r-1 \\
\neq 0, \text { for } j=n-r .
\end{array}\right.
$$

A more general definition of quasi-orthogonality is given in [10, p.64] for order 1 and in [11] for any order. We say that a polynomial $P_{n}$ of exact degree $n \geq r$, $n=0,1, \ldots, N$, where $N$ may be infinite, is discrete quasi-orthogonal of order $r$ with $\rho_{i}$ being the values of the weight at the points $x_{i}, i=0,1, \ldots, N-1$, if

$$
\sum_{i=0}^{N-1}\left(x_{i}\right)^{j} P_{n}\left(x_{i}\right) \rho_{i}\left\{\begin{array}{l}
=0, \text { for } j=0,1, \ldots, n-r-1 \\
\neq 0, \text { for } j=n-r .
\end{array}\right.
$$

The Meixner polynomials $M_{n}(x ; \beta, c)$ are orthogonal on $(0, \infty)$ for $0<c<1$, $\beta>0$ and as $\beta$ decreases below 0 , the zeros of $M_{n}(x ; \beta, c)$ depart from the interval of orthogonality $(0, \infty)$. We prove the quasi-orthogonality of these polynomials in the following theorem.

Theorem 3 The polynomials $M_{n}(x ; \beta-k, c)$ with $0<c<1,0<\beta<1$ and $k=1,2, \ldots, n-1$ are quasi-orthogonal of order $k$ with respect to the weight function $\frac{c^{x}(\beta)_{x}}{x !}$ on $(0, \infty)$.

Proof The recurrence relation (cf. [31, p.71 (2)])

$$
M_{n}(x ; \beta, c)=n M_{n-1}(x ; \beta, c)+M_{n}(x ; \beta-1, c)
$$

shows that $M_{n}(x ; \beta-k, c)$ can be expressed as a linear combination of $M_{n}(x ; \beta, c)$, $M_{n-1}(x ; \beta, c), \ldots, M_{n-k}(x ; \beta, c)$ and, since $\beta>0$, it follows from (3) that

$$
\sum_{x=0}^{\infty} x^{j} M_{n}(x ; \beta-k, c) \frac{c^{x}(\beta)_{x}}{x !}=0 \text { for } j=0,1, \ldots, n-k-1 .
$$

Remark 4 By a change of variable, the result in Theorem 3 can be written as that the polynomials $M_{n}(x ; \beta, c)$ are quasi-orthogonal of order $k$ on $(0, \infty)$, for $0<c<1$ and $-k<\beta<-k+1, k=1,2, \ldots, n-1$ with respect to the weight function $\frac{c^{x}(\beta+k)_{x}}{x !}$.

The zeros of quasi-orthogonal polynomials are not necessarily all in the interval of orthogonality, but we can say the following from [8, Theorem 2].

Corollary 5 The Meixner polynomials $M_{n}(x ; \beta, c)$, with $0<c<1,-k<\beta<$ $-k+1$ have at least $n-k$ zeros in $(0, \infty)$ when $k=1,2, \ldots, n-1$. 
In order to specify the location of the remaining single zero of $M_{n}(x ; \beta-1, c)$, $0<c<1,0<\beta<1$ where we have quasi-orthogonality of order 1 , we consider the monic polynomials $\tilde{M}_{n}(x ; \beta, c)=\left(\frac{c}{c-1}\right)^{n} M_{n}(x ; \beta, c)$.

Theorem 6 If $0<c<1$ and $0<\beta<1$, then the smallest zero of $M_{n}(x ; \beta-1, c)$ (or equivalently $\tilde{M}_{n}(x ; \beta-1, c)$ ) is negative.

Proof The recurrence relation (19) can be written as

$$
\tilde{M}_{n}(x ; \beta-1, c)=\tilde{M}_{n}(x ; \beta, c)-n\left(\frac{c}{c-1}\right) \tilde{M}_{n-1}(x ; \beta, c)
$$

and according to [23, Theorem 4$]$ we have to show that $n\left(\frac{c}{c-1}\right)<\frac{\tilde{M}_{n}(0 ; \beta, c)}{\tilde{M}_{n-1}(0 ; \beta, c)}<0$ which follows immediately from (18).

Joulak's results (cf. [23, Theorems 8, 9]) also gives some information about the location of the zeros when we have quasi-orthogonality of order 2 .

Theorem 7 If $0<c<1,0<\beta<1$ and $n>\frac{\beta-2}{c-1}$ then all the zeros of $M_{n}(x ; \beta-2, c)$ are nonnegative and simple.

Proof Iterating (20) we obtain

$$
\tilde{M}_{n}(x ; \beta-2, c)=\tilde{M}_{n}(x ; \beta, c)-2 n\left(\frac{c}{c-1}\right) \tilde{M}_{n-1}(x ; \beta, c)+b_{n} \tilde{M}_{n-2}(x ; \beta, c)
$$

where $b_{n}=n(n-1)\left(\frac{c}{c-1}\right)^{2}$. Replacing $n$ by $n-1$ in (11) yields

$$
\tilde{M}_{n}(x ; \beta, c)=\left(x-B_{n-1}\right) \tilde{M}_{n-1}(x ; \beta, c)-\left(\frac{c}{c-1}\right) C_{n-1} \tilde{M}_{n-2}(x ; \beta, c) .
$$

From [23, Theorem 8] all the zeros of $\tilde{M}_{n}(x ; \beta-2, c)$ are real and simple if $b_{n}<\left(\frac{c}{c-1}\right) C_{n-1}$ which gives the condition $n>\frac{\beta-2}{c-1}$. Furthermore, the smallest zero (and hence all of the zeros) of $M_{n}(x ; \beta-2, c)$ is nonnegative if and only if (cf. [23, Theorem 9])

$$
\frac{\tilde{M}_{n}(0 ; \beta, c)}{\tilde{M}_{n-2}(0 ; \beta, c)}-2 n\left(\frac{c}{c-1}\right) \frac{\tilde{M}_{n-1}(0 ; \beta, c)}{\tilde{M}_{n-2}(0 ; \beta, c)}+n(n-1)\left(\frac{c}{c-1}\right)^{2} \geq 0 .
$$

It follows from (18) that the left-hand side simplifies to $\left(\frac{c}{c-1}\right)^{2}(1-\beta)(2-\beta)$, which is positive by the assumptions. This completes the proof.

Analogous results can be obtained for the polynomials $M_{n}(x ; \beta, c), \beta<0$ and $c>1$. 
4 The zeros of $K_{n}(x ; p, N), 0<p<1$ and $n=N+1, N+2, \ldots$

We now turn our attention to the zeros of the polynomials $K_{n}(x ; p, N), 0<p<$ 1 and $n, N \in \mathbb{N}$ for degrees higher than what follows from orthogonality, i.e. for $n=N+k, k \in \mathbb{N}$. Note that in this case the coefficient $C_{n}=n(1-p)(N-n+1)$ in the three term recurrence relation (cf. $[24,(9.11 .3)]$ )

$$
\begin{aligned}
x K_{n}(x ; p, N) & =p K_{n+1}(x ; p, N)+B_{n} K_{n}(x ; p, N)+C_{n} K_{n-1}(x ; p, N) \\
K_{0} & =1, \quad K_{-1}=0,
\end{aligned}
$$

becomes nonpositive, thus the polynomials are non-orthogonal on the real line. In the case $n=N+1$ we have $C_{n}=0$ and a degenerate version of Favard's theorem ensures a non-standard ( $\Delta$-Sobolev) orthogonality (cf. [13, Theorem $2.2])$. This case is also related to Sylvester type determinants, first studied by J. Sylvester (cf. [34]) and more recently by R. Askey and G. Wilson (cf. [3]) in connection with the $q$-Racah polynomials. The connection of orthogonal polynomials, specifically Krawtchouk polynomials, with tridiagonal matrices whose entries come from the recurrence coefficients of discrete orthogonal polynomials is made explicit in [4] and [19].

Lemma 8 (cf. $[35, p .36])$ Let $0<p<1$ and $N$ a positive integer, the polynomials $K_{N+1}(x ; p, N)=(x)(x-1) \ldots(x-N)\left(\frac{1}{p}\right)^{N+1}$ and have $N+1$ real zeros $x=0,1, \ldots, N$.

Corollary 9 For $0<p<1$ and $N$ a positive integer, the polynomial $K_{N+2}(x ; p, N)$ has $N+2$ real zeros

$$
x=0,1, \ldots, N, N+1-p(N+2) .
$$

Proof Letting $n=N+2$ in (10) we obtain

$$
K_{N+2}(x ; p, N)=K_{N+1}(x, p, N)\left(N+2+\frac{x-(N+1)}{p}\right)
$$

which yields the stated result.

Corollary 10 For $0<p<1$ and $N$ a positive integer, the polynomial $K_{N+3}(x ; p, N)$ has at least $N+1$ real zeros $x=0,1, \ldots, N$. Furthermore, the remaining two zeros will be real and distinct when

$$
0<p<\frac{1}{2}\left(1-\sqrt{\frac{N+2}{N+3}}\right) \text { or } \frac{1}{2}\left(1+\sqrt{\frac{N+2}{N+3}}\right)<p<1 .
$$

Proof It follows from (10) that $K_{N+3}(x ; p, N)=K_{N+1}(x ; p, N) p_{2}(x)$, where

$$
\begin{aligned}
p_{2}(x)= & \frac{1}{p^{2}}\left(x^{2}+(6 p+2 N p-3-2 N) x+(N+3)(N+2) p^{2}-\right. \\
& 2(N+1)(N+3) p+(N+1)(N+2)) .
\end{aligned}
$$


The zeros of the quadratic $p_{2}(x)$ are real and distinct when the discriminant $\frac{1}{p^{4}}\left(1-4 p(N+3)+4 p^{2}(N+3)\right)$ is positive and this yields the stated result.

It is difficult to determine the exact location of the zeros in the general case $K_{n}(x ; p, N), n=N+k, k \in \mathbb{N}$. There are only $N+1$ or $N+2$ real zeros, but one can use (10) to consider the polynomials $K_{n}(x ; p,-N)$ for $0<p<1$, $N \in \mathbb{N}$ instead.

In general, it suffices to investigate the zeros of $M_{n}(x ; \beta, c)$ for $\beta>0$ when $-1 \leq c<0$ (or $c \leq-1$ ), because it follows from the symmetry relation (9) that if $x$ is a zero of $M_{n}(x ; \beta, c)$ then $-\beta-x$ is a zero of $M_{n}\left(x ; \beta, \frac{1}{c}\right)$. Taking into consideration the complex conjugate pairs, geometrically it means that the zeros of $M_{n}\left(x ; \beta, \frac{1}{c}\right)$ are the mirror image of the zeros of $M_{n}(x ; \beta, c)$ with respect to the axis $\operatorname{Re} x=-\beta / 2$ when $\beta>0$ and $c<0$.

Figure 1 shows the zeros of $M_{n}(x ; \beta, c)$ when $n=10, \beta=8.2$ for different values of $c<0$, clearly illustrating the symmetry with respect to $\operatorname{Re} x=-4.1$.

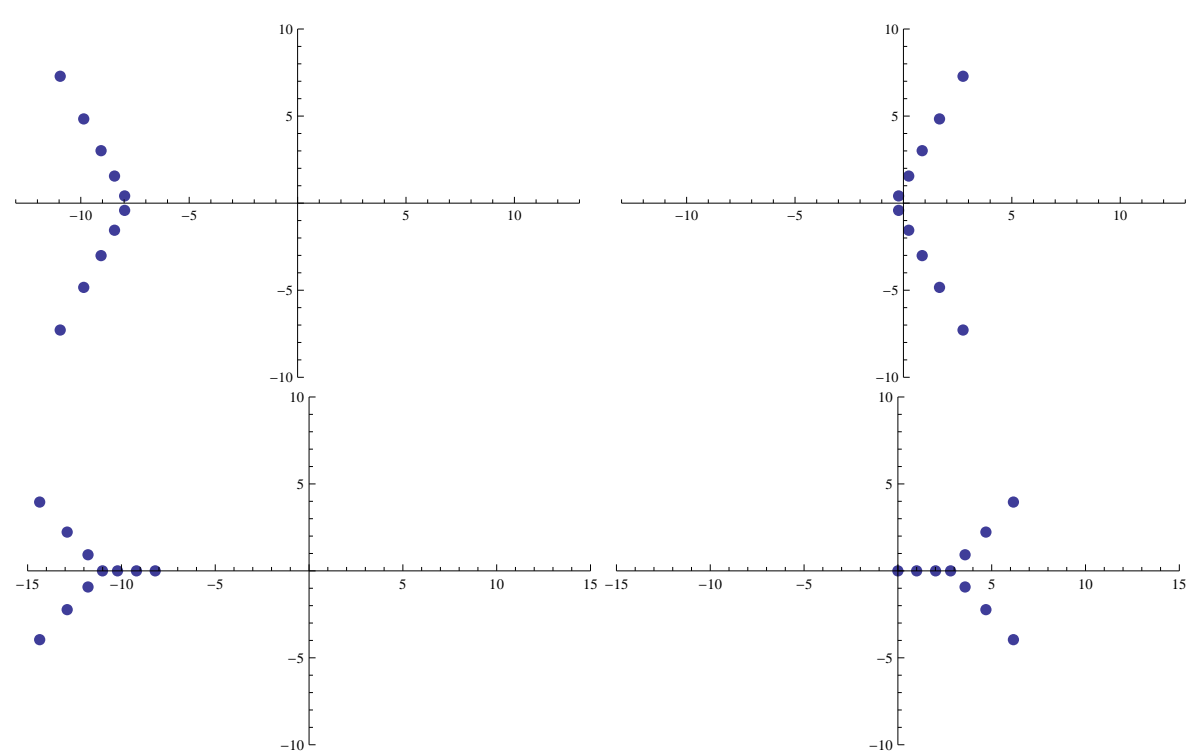

Fig. 1 The zeros of $M_{10}(x ; 8.2,-4), \quad M_{10}(x ; 8.2,-0.25), \quad M_{10}(x ; 8.2,-15.667)$ and $M_{10}(x ; 8.2,-0.064)$ clockwise.

The numerical examples show that the zeros of polynomials $M_{n}(x ; \beta, c), \beta>0$, $c<0$ seem to lie on rays starting from the $x$ axis and for the special case $c=$ -1 , all the zeros of polynomials $M_{n}(x ; \beta, c), \beta>0$ lie on the line $\operatorname{Re} x=-\beta / 2$. This special case when $c=-1$ is illustrated in Figure 2. 


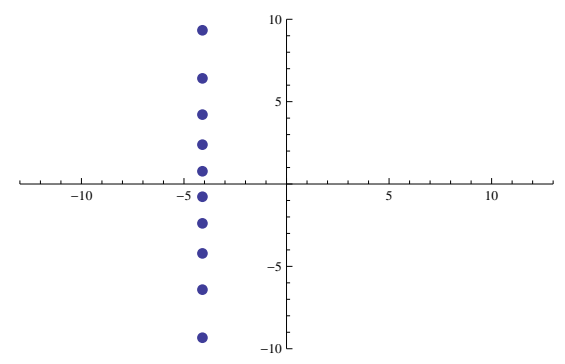

Fig. 2 The zeros of $M_{10}(x ; 8.2,-1)$

The asymptotic distribution of the zeros of $M_{n}(x ; \beta, c), \beta>0, c<0$ as $n \rightarrow \infty$, (after the necessary rescaling) could possibly be proved using the complex orthogonality (cf. [12]) and potential theoretical methods or a Riemann-Hilbert approach, as was done in the case of Jacobi polynomials for non-standard parameters (cf. [27], [29]).

Remark 11 Letting $a=-n, b=-x, c=-N-1$ and $z=\frac{1}{p}$ in the contiguous relation (cf. [31, p.71 (3)])

$$
\begin{aligned}
& (a+(b-c) z){ }_{2} F_{1}(a, b ; c ; z) \\
& =a(1-z){ }_{2} F_{1}(a+1, b ; c ; z)-\frac{(c-a)(c-b) z}{c}{ }_{2} F_{1}(a, b ; c+1 ; z)
\end{aligned}
$$

we obtain

$$
\begin{aligned}
& (x-N-1) K_{n}(x ; p, N) \\
& =n(n-N-2)(1-p) K_{n-1}(x ; p, N+1)+(x+n p-N-1) K_{n}(x ; p, N+1) .
\end{aligned}
$$

Using this it is easy to show by induction that

$(x-N-1)(x-N-2) \ldots(x-N-j) K_{n}(x ; p, N)=\sum_{i=0}^{j} q_{i}(x) K_{n-i}(x ; p, N+j)$,

where $q_{i}(x)$ are polynomials of degree $j-i$. From this it follows $(c f .[8, p .160])$ that $(x-N-1)(x-N-2) \ldots(x-N-k) K_{n}(x ; p, N)($ with $n=N+k)$ is quasi-orthogonal of degree $N+2 k$ and order $2 k$. However, unlike in Section 3 this does not lead to new information about real zeros, since these are already guaranteed by formula (10) and Lemma 8.

Remark 12 For the sake of completeness we note that similarly one can prove that for $c<0$ and $0<-\beta<n-1$, where $\beta$ is non-integer, the polynomial $(x+\beta-1)(x+\beta-2) \ldots(x+\beta-(n-\lfloor-\beta\rfloor-1)) M_{n}(x ; \beta, c)$ is quasi-orthogonal of degree $2 n-\lfloor-\beta\rfloor-1$ and order $2 n-2\lfloor-\beta\rfloor-2$. 


\section{The zeros of $M_{n}(x ; \beta, c), c \rightarrow 0$ and $\beta \in \mathbb{R}$}

Next, we consider $M_{n}(x ; \beta, c)$ when $\beta \in \mathbb{R}$ and prove that when $c \rightarrow 0$ all the zeros of $M_{n}(x ; \beta, c), n=1,2, \ldots$ approach non-negative integer values. Note that this theorem holds for any $\beta \in \mathbb{R}$ which implies that when $-\beta=N$, $N \in \mathbb{N}$ and $c<0$, the zeros of the Krawtchouk polynomials $K_{n}\left(x ; \frac{c}{c-1}, N\right)$ approach the mass points of the weight function as $c \rightarrow 0$.

Theorem 13 For $n \in \mathbb{N}$ and $\beta \in \mathbb{R}$, the $n$ zeros of the polynomial $M_{n}(x ; \beta, c)$ approach the points $x=0,1, \ldots, n-1$ when $c \rightarrow 0$.

$$
\begin{aligned}
& \text { Proof } \\
& M_{n}(x ; \beta, c) \\
& =(\beta)_{n}+(\beta+1)_{n-1}(-n)(-x)\left(1-\frac{1}{c}\right)+\cdots+ \\
& \quad \frac{(\beta+n-1)(-n)_{n-1}(-x)_{n-1}\left(1-\frac{1}{c}\right)^{n-1}}{(n-1) !}+(x)(x-1) \ldots(x-n+1)\left(1-\frac{1}{c}\right)^{n} .
\end{aligned}
$$

For any $n \in \mathbb{N}$ the function

$$
\begin{aligned}
c^{n} M_{n}(x ; \beta, c) & =c^{n}(\beta)_{n}+\cdots+\frac{c(\beta+n-1)(-n)_{n-1}(-x)_{n-1}(c-1)^{n-1}}{(n-1) !} \\
& +(x)(x-1) \ldots(x-n+1)(c-1)^{n}
\end{aligned}
$$

regarded as an $n$th degree polynomial in $x$ with real parameters $\beta$ and $c$ has the same zeros as $M_{n}(x ; \beta, c)$. Since

$$
\lim _{c \rightarrow 0} c^{n} M_{n}(x ; \beta, c)=(x)(x-1) \ldots(x-n+1)(-1)^{n},
$$

the zeros of $c^{n} M_{n}(x ; \beta, c)$ and hence the zeros of $M_{n}(x ; \beta, c)$ tend to the zeros of $x(x-1)(x-2) \ldots(x-n+1)$, which is to say $x=0,1,2, \ldots, n-1$.

This theorem implies that for sufficiently small $c$ all the zeros of $M_{n}(x ; \beta, c)$ are real. An analogous result can be proved for Charlier polynomials.

Acknowledgements The authors would like to thank the anonymous referee for pertinent observations and remarks. The first two authors would like to thank the Institute for Biomathematics and Biometry at the Helmholtz Zentrum München for their support during a research visit.

\section{References}

1. M. Álvarez de Morales, T. Pérez, M. Piñar \& A. Ronveaux, Non-standard orthogonality for Meixner polynomials, Electron. Trans. Numer. Anal. 9 (1999), 1-25.

2. R. Álvarez-Nodarse \& J. S. Dehesa, Distributions of zeros of discrete and continuous polynomials from their recurrence relation, Appl. Math. Comput. 128 (2-3) (2002), 167190.

3. R. Askey \& J. Wilson, A set of orthogonal polynomials that generalize the Racah coefficients or 6-j symbols, SIAM J. Math. Anal. 10 (1979), 1008-1016. 
4. R. Askey, Evaluation of Sylvester Type Determinants Using Orthogonal Polynomials, Advances in Analysis: Proc. 4th Internat. ISAAC Congress, (H.G.W. Begehr et al, eds.), World Scientific (2005), 1-16.

5. F. V. Atkinson, Discrete and continuous boundary problems, Mathematics in Science and Engineering, Academic Press, New York (1964).

6. P. Braenden, On operators on polynomials preserving real-rootedness and the NeggersStanley conjecture, J. Algebr. Comb. 20 (2004), 119-130.

7. F. Brenti, Log-concave and unimodal sequences in algebra, combinatorics, and geometry: an update, Jerusalem Combinatorics '93, Contemporary Mathematics 178, Amer. Math. Soc., Providence, (1994), 71-89.

8. C. Brezinski, K. A. Driver \& M. Redivo-Zaglia, Quasi-orthogonality with applications to some families of classical orthogonal polynomials, Appl. Numer. Math. 48 (2004), $157-168$.

9. L. Chihara \& D. Stanton, Zeros of generalized Krawtchouk polynomials, J. Approx. Theory 60 (1), (1990), 43-57.

10. T. S. Chihara, An Introduction to orthogonal polynomials, Gordon and Breach (1978).

11. T. S. Chihara, On quasi-orthogonal polynomials, Proc. Amer. Math. Soc. 8 (1957), 765-767.

12. R. S. Costas-Santos \& J. F. Sánchez-Lara, Extensions of discrete classical orthogonal polynomials beyond the orthogonality, J. Comput. Appl. Math. 225 (2009), 440-451.

13. R. S. Costas-Santos \& J. F. Sánchez-Lara, Orthogonality of $q$-polynomials for nonstandard parameters, J. Approx. Theory 163(9) (2011), 1246-1268.

14. P. D. Dragnev \& E. B. Saff, Constrained energy problems with applications to orthogonal polynomials of a discrete variable, J. Anal. Math. 72 (1997), 223-259.

15. P. D. Dragnev \& E. B. Saff, A problem in potential theory and zero asymptotics of Krawtchouk polynomials, J. Approx. Theory 102(1) (2000), 120-140.

16. K. Driver \& K. Jordaan, Convergence of ray sequences of Padé approximants to ${ }_{2} F_{1}(a, 1 ; c ; z)$, Quaest. Math. 25 (2002), 1-7.

17. K. Driver, K. Jordaan \& A. Martínez-Finkelshtein, Pólya frequency sequences and real zeros of some ${ }_{3} F_{2}$ polynomials, J. Appl. Math. Anal. Appl. 332(2) (2007), 1045-1055.

18. I. B. Grafova \& B. M. Grafov, Meixner wavelet transform: A tool for studying stationary discrete-time stochastic processes, Russ. J. Electrochem. 39(2) (2003), 130-133.

19. O. Holtz, Evaluation of Sylvester Type Determinants using Block-triangularization, Advances in Analysis: Proc. 4th Internat. ISAAC Congress, (H.G.W. Begehr et al, eds.), World Scientific (2005), 395-405.

20. M. E. H. Ismail \& P. Simeonov, Strong asymptotics for Krawtchouk polynomials, J. Comput. Appl. Math. 100(2) (1998), 121-144.

21. X. Jin \& R. Wong, Asymptotic formulas for the zeros of the Meixner polynomials, $J$. Approx. Theory 96 (1999), 281-300.

22. C. Jordan, Sur une série de polynomes, Proc. Lond. Math. Soc. 20(2) (1920), 297-325.

23. H. Joulak, A contribution to quasi-orthogonal polynomials and associated polynomials, Appl. Numer. Math. 54(1)(2005), 65-78.

24. R. Koekoek, P. A. Lesky \& R. F. Swarttouw, Hypergeometric orthogonal polynomials and their q-analogues. Springer Monogr. Math., Springer Verlag, Berlin (2010).

25. T. H. Koornwinder, Krawtchouk polynomials, a unification of two different group theoretic interpretations, SIAM J. Math. Anal. 13 (1982), 1011-1023.

26. I. V. Krasovsky, Asymptotic distribution of zeros of polynomials satisfying difference equations, J. Comput. Appl. Math. 150(1) (2003), 57-70.

27. A. B. J. Kuijlaars \& A. Martínez-Finkelshtein, Strong asymptotics for Jacobi polynomials with varying nonstandard parameters, J. Anal. Math. 94 (2004), 195-234.

28. R. J. Levit, The zeros of the Hahn polynomials, SIAM Rev. 9(2) (1967), 191-203.

29. A. Martínez-Finkelshtein, P. Martínez-González \& R. Orive, Zeros of Jacobi polynomials with varying non-classical parameters, Special functions. Proc. of the internat. workshop on special functions - asymptotics, harmonic analysis and mathematical physics, (Charles Dunkl et al eds.), Hong Kong, China, June 21-25, 1999. Singapore: World Scientific, (2000), 98-113.

30. M. Porter, On the roots of functions connected by a linear recurrent relation of the second order, Annals of Math. 3(2) (1902), 55-70. 
31. E. D. Rainville, Special Functions. The Macmillan Company, New York (1960).

32. V. N. Sorokin, On multiple orthogonal polynomials for discrete Meixner measures, $S b$. Math. 201(10) (2010), 1539-1561.

33. R. Stanley, Log-concave and unimodal sequences in algebra, combinatorics and geometry, Ann. NY. Acad. Sci. 576 (1989), 500-534.

34. J. J. Sylvester, Théorème sur les déterminants, Nouv. Ann. de Math. XIII (1854), 305, Reprinted in The collected mathematical papers of JJ Sylvester vol II, 28.

35. G. Szegö, Orthogonal Polynomials, Fourth ed., Amer. Math. Soc., Coll. Publ. vol. 23, Providence, RI, (1975). 